\title{
MOBILIZAÇÃO PRECOCE NA UNIDADE DE TERAPIA INTENSIVA EM PACIENTES COM VENTILAÇÃO MECÂNICA: REVISÃO SISTEMÁTICA
}

\author{
EARLY MOBILIZATION IN THE INTENSIVE CARE UNIT IN PATIENTS WITH \\ MECHANICAL VENTILATION: SYSTEMATIC REVIEW
}

\author{
Suélen Amâncio da Rosa ${ }^{1}$, Fernanda Emanuelle Viomar Rocha ${ }^{2}$, \\ Alana Zentil Buss ${ }^{3}$, Bruna Aparecida Metinoski Bueno ${ }^{4}$, \\ Giovana Frazon de Andrade ${ }^{5}$ Ana Carolina Dorigoni Bini ${ }^{6}$
}

\section{RESUMO}

Objetivo: Sintetizar as evidências científicas da eficácia da mobilização precoce em pacientes internados em UTI com uso de ventilação mecânica por meio de revisão sistemática. Métodos: Trata-se de revisão sistemática sobre a mobilização precoce em pacientes com uso de ventilação mecânica, seguindo o protocolo PRISMA. Foram incluídos artigos no idioma inglês e português, e publicados entre 2016 e 2020. Resultados: A busca identificou 1.012 artigos, sendo 344 no Google Scholar, 25 na Cochrane, 3 no Lilacs, 8 na SciELO, 256 na PubMed, 16 na Medline, 360 na Scopus. Após análise criteriosa, foram selecionados pelo título 29 artigos, e destes, foram elegíveis para este estudo 19 artigos que apresentavam os critérios de inclusão. Conclusão: Com base nos resultados obtidos, concluiu-se que a mobilização precoce na UTI apresentou um impacto significativamente positivo nos resultados funcionais dos pacientes, como também na redução do tempo de internação e o tempo de ventilação mecânica em pacientes críticos.

Palavras-chave: Fisioterapia, Mobilização precoce, Ventilação mecânica, Unidade de Terapia Intensiva.

\section{ABSTRACT}

Objective: Synthesize the scientific evidence of the effectiveness of early mobilization in patients admitted to the ICU using mechanical ventilation through a systematic review. Methods: This is a systematic review of early mobilization in patients using mechanical ventilation, following the PRISMA protocol. Articles in English and Portuguese were included, and published between 2016 and 2020. Results: The search identified 1,012 articles, 344 in Google Scholar, 25 in Cochrane, 3 in Lilacs, 8 in SciELO, 256 in PubMed, 16 in Medline, 360 in Scopus. After careful analysis, 29 articles were selected by title, and of these, 19 articles that met the inclusion criteria were eligible for this study. Conclusion: Based on the results obtained, it was concluded that early mobilization in the ICU had a significantly positive impact

1 Autor: Suélen Amâncio da Rosa, graduanda em Fisioterapia pela Universidade Estadual do Centro Oeste (UNICENTRO). E-mail: suelen_vieira98@hotmail.com

2 Coautor: Fernanda Emanuelle Viomar Rocha, graduanda em Fisioterapia pela Universidade Estadual do Centro Oeste (UNICENTRO).E-mail: fevrocha@gmail.com

3 Coautor: Alana Zentil Buss, graduanda em Fisioterapia na Universidade Estadual do Centro Oeste (UNICENTRO). E-mail: alanazb@hotmail.com

4 Coautor: Bruna Aparecida Metinoski Bueno, graduanda em Fisioterapia na Universidade Estadual do Centro Oeste (UNICENTRO).E-mail: bruna_metinoski@hotmail.com

5 Coorientador: Giovana Frazon de Andrade, docente no Departamento de Fisioterapia da Universidade Estadual do Centro-Oeste (UNICENTRO). E-mail: giovanafrazon@hotmail.com

6 Orientador: Ana Carolina Dorigoni Bini, docente do Departamento de Fisioterapia da Universidade Estadual do Centro Oeste (UNICENTRO).E-mail: ana.carolina.db@hotmail.com 
on the functional outcomes of patients, as well as on the reduction of hospital stay and mechanical ventilation time in critically ill patients.

Keywords: Early ambulation; Physical therapy, Mechanical ventilation, Intensive Care Units.

\section{INTRODUÇÃO}

A ventilação mecânica (VM) é uma terapia de manutenção da vida para o tratamento de pacientes com insuficiência respiratória aguda, sendo uma modalidade muito comum nas Unidades de Terapia Intensiva (UTI) (SLUTSKY, 2015). Devido à baixa mobilidade durante o repouso no leito, as pessoas internadas em UTI estão frequentemente sujeitas a danos relacionados à imobilidade em diversos sistemas (SCHUJMANN et al., (2019). A fraqueza muscular generalizada é uma complicação frequente que se desenvolve durante o período de internação na UTI, e é rotulada como "fraqueza adquirida na unidade de terapia intensiva" (FAUTI), podendo afetar os músculos periféricos e respiratórios (HERMANS; VAN DEN BERGHE, 2015) e está associada ao aumento do risco de mortalidade, maior tempo de internação na UTI e enfermaria, diminuição da pontuação Medical Research Council (MRC) e aumento dos custos relacionados à saúde (ZANG et al., 2018). A preservação da função muscular esquelética é um fator de relevância fundamental no paciente crítico e a conservação da massa muscular é um grande desafio (GODOY et al., 2015).

Como forma de minimizar ou reverter as disfunções causadas em pacientes que foram submetidos à VM, a mobilização precoce (MP) desempenha um importante papel no processo de preservação e recuperação funcional (MATOS et al., 2016). A MP engloba atividades progressivas, como exercícios passivos, assistidos e ativos no leito, seguido do sentar à beira do leito, transferência para a poltrona, ortostatismo, marcha estacionária e deambulação (SOUZA et al., 2019). As complicações da perda de força e massa muscular são passíveis de prevenção e recuperação e o fisioterapeuta tem um importante papel neste sentido, atuando na melhora da capacidade funcional dos pacientes, assim como na restauração da independência respiratória e física, diminuindo os riscos de complicações (BIANCHI, 2016; ROTTA et al., 2018).

Na última década, houve um aumento de evidências científicas apoiando a segurança, viabilidade e benefícios funcionais da utilização da MP em pacientes críticos a partir das primeiras 48 horas da instituição da VM (AQUIM et al., 2019), sendo uma escolha terapêutica crescente nos centros de tratamento (COUTINHO et al., 2016). A equipe multidisciplinar é a responsável por identificar as indicações e as contraindicações para realizar MP, porém, cabe ao fisioterapeuta estabelecer o melhor modelo de intervenção, intensidade, periodicidade, continuidade ou interrupção, verificando todos os critérios de segurança.

A redução dos fatores de risco em conjunto com a MP podem beneficiar substancialmente os pacientes em risco de FAUTI (KRAMER, 2017), tendo efeitos significativos no transporte de oxigênio, 
manutenção da força muscular e mobilidade articular, função pulmonar e desempenho do sistema respiratório (DANTAS et al., 2012). Isso faz com que reduza a duração da VM e o tempo de permanência no hospital e na UTI, melhorando a função física na alta hospitalar e reduzindo as taxas de readmissão e mortalidade hospitalares em longo prazo (FONTELA et al., 2018). Diante do exposto, o objetivo do presente estudo foi sintetizar as evidências científicas da eficácia da mobilização precoce em pacientes internados em UTI com o uso de ventilação mecânica por meio de revisão sistemática.

\section{METODOLOGIA}

Trata-se de revisão sistemática elaborada seguindo as recomendações do protocolo Preferred Reporting Items of Systematic Reviews and Meta-Analyses PRISMA(GALVÃO; PANSANI; HARRAD, 2015). A busca por artigos foi realizada nas bases de dados Google Acadêmico, Cochrane, MedLine, LILACS, SciELO, PEdro, PubMed e Scopus. Os descritores foram "ventilação mecânica" AND "mobilização precoce" AND "fisioterapia", e "mechanical ventilation" AND "early ambulation" AND "physical therapy”. Os critérios de inclusão foram artigos originais (ensaios clínicos, estudos de caso, relatos de caso e estudos retrospectivos) sobre mobilização precoce em pacientes adultos com o uso de ventilação mecânica, no idioma inglês ou português, e publicados entre 2016 e 2020, sendo essa data escolhida para a obtenção de artigos mais atuais sobre o assunto. Foram excluídos artigos de revisão bibliográfica, realizados com população infantil, pacientes oncológicos ou publicações anteriores à 2016.

Todo o processo de seleção e revisão dos artigos identificados por meio da estratégia de busca foi realizado independentemente por dois pesquisadores, que confrontaram seus resultados em cada uma das etapas. Inicialmente, excluiu-se os artigos duplicados, em seguida, avaliou-se o tema pela leitura dos títulos e resumos. Após, realizava-se a leitura na íntegra, verificando se atendia aos critérios de inclusão.

Os ensaios clínicos selecionados foram avaliados pela escala Physiotherapy Evidence Database (PEDro), que avalia a qualidade metodológica por meio de 11 itens preestabelecidos, com escores de 0 a 10. Nesta etapa, dois pesquisadores independentes aplicaram a escala de qualidade PEDro nos artigos selecionados, confrontando os resultados e resolvendo as discrepâncias por consenso, chegando à pontuação final. A pontuação da escala não foi utilizada como critério de inclusão/exclusão dos artigos, mas como indicador de evidência científica.

\section{RESULTADOS}

A busca identificou 1.012 artigos, sendo 344 no Google Scholar, 25 na Cochrane, 3 no Lilacs, 8 na SciELO, 256 na PubMed, 16 na Medline e 360 na Scopus. Após análise criteriosa, foram selecio- 
nados pelo título 29 artigos e, destes, foram elegíveis para este estudo 19 artigos que apresentavam os critérios de inclusão (Figura 1).

Figura 1 - Fluxograma dos artigos incluídos

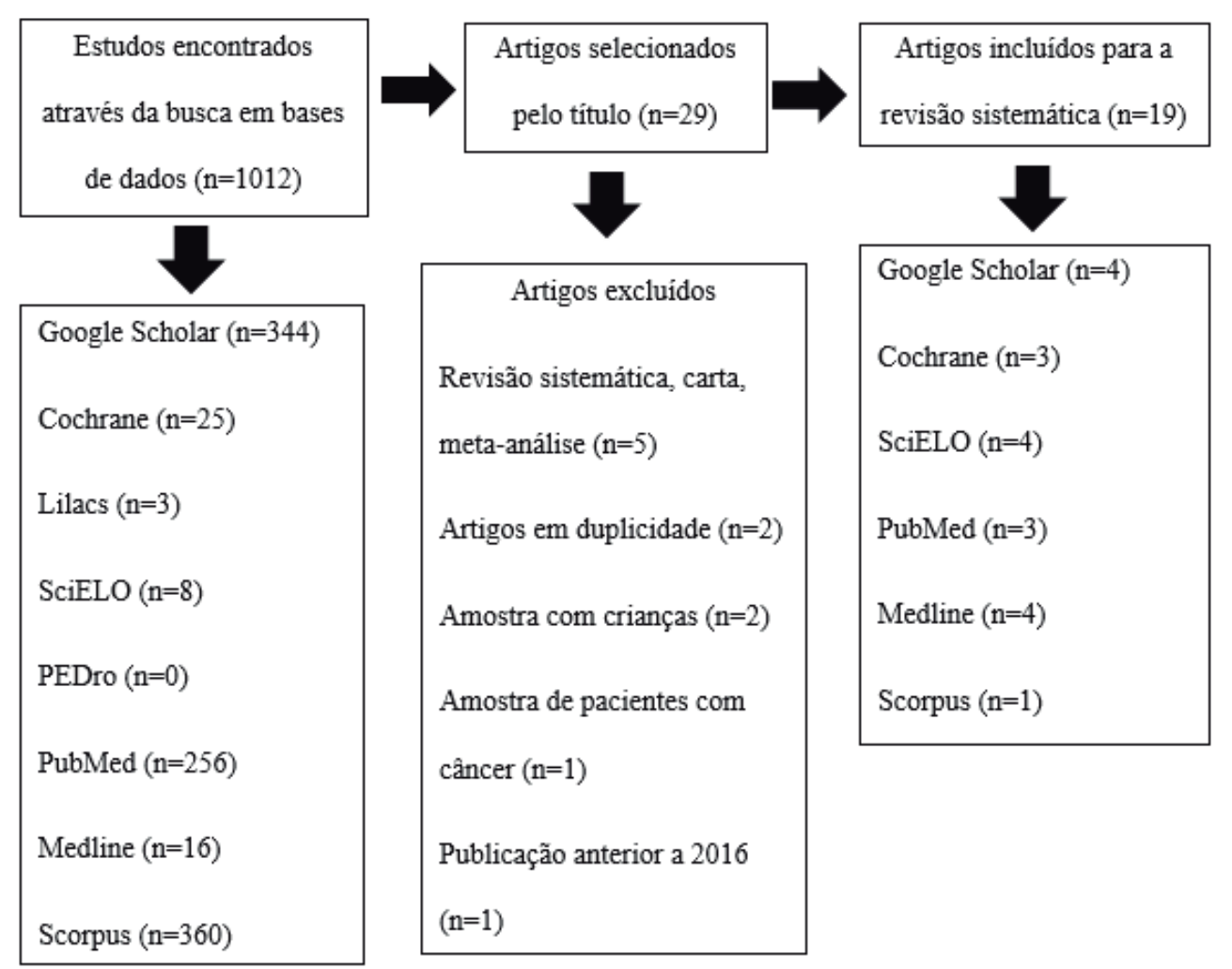

Fonte: Construção dos autores.

Na Tabela 1 são apresentadas as características dos estudos incluídos nesta revisão. Entre os dezenove artigos analisados, nove são ensaios clínicos, dois são estudos observacionais, um estudo retrospectivo, um estudo de prevalência e dois estudos de coorte. O tamanho das amostras variou de 14 à 541 participantes, totalizando 2.397 integrantes, sendo a idade mínima de inclusão de 16 anos, com população de homens e mulheres. Foram encontrados estudos realizados em hospitais do Brasil, Suíça, Áustria, Alemanha, Estados Unidos, Inglaterra, Japão, Austrália, Nova Zelândia e Macedônia. A inclusão do uso da MP em indivíduos sob VM foi observada em todos os estudos, exceto no estudo de Liu et al. (2018) onde foi incluído pacientes com VM e com oxigenação por membrana extracorpórea. Em relação ao protocolo de MP, alguns autores compararam diferentes tempos de início da MP, fisioterapia convencional e adição de cicloergômetro, exercícios de transferência e deambulação, treinamento precoce e progressivo de resistência, atividades funcionais ativas e restrição ou não ao leito. Os resultados apresentaram variação, desde diferença significativa ou não do tempo de VM, preservação de mobilidade, aumento de força periférica e diminuição do tempo de internação na UTI. 
Tabela 1 - Descrição dos estudos incluídos nesta revisão.

\begin{tabular}{|c|c|c|c|c|c|}
\hline & Referência & Tipo de estudo & Amostra & Protocolo de Mobilização Precoce & Resultados \\
\hline 1 & $\begin{array}{l}\text { ABRUZZI } \\
\text { et al. (2017) }\end{array}$ & $\begin{array}{l}\text { Ensaio clínico } \\
\text { randomizado }\end{array}$ & $n=120$ & $\begin{array}{l}\text { G1: atendidos entre } 0-12 \mathrm{~h} \text {; G2: entre } \\
13 \text { e } 24 \mathrm{~h} \text { e } \\
\text { G3: atendidos após } 24 \mathrm{~h} \text {. Duração de } \\
30 \text { min, } 2 \mathrm{x} \text { ao dia. }\end{array}$ & $\begin{array}{l}\text { O G1 e G2 apresentaram menor } \\
\text { tempo de VM e redução no tempo } \\
\text { de hospitalização. }\end{array}$ \\
\hline 2 & BIANCHI (2016) & $\begin{array}{l}\text { Ensaio clínico } \\
\text { randomizado }\end{array}$ & $n=42$ & $\begin{array}{l}\text { GC: fisioterapia } 1 x \text { por dia por } 30 \\
\text { min. } \\
\text { GI: realizou adicionalmente o ci- } \\
\text { cloergômetro } 1 x \text { por dia por } 20 \mathrm{~min} \text {. }\end{array}$ & $\begin{array}{l}\text { Preservação da mobilidade } \\
\text { diafragmática. Sem diferenças no } \\
\text { tempo de VM, sucesso na extuba- } \\
\text { ção e óbitos. }\end{array}$ \\
\hline 3 & $\begin{array}{l}\text { CAVALCANTE } \\
\text { et al. (2018) }\end{array}$ & $\begin{array}{l}\text { Observacional, } \\
\text { transversal e } \\
\text { quantitativo }\end{array}$ & $n=15$ & $\begin{array}{l}\text { Protocolo de MP consistiu em } 15 \\
\text { repetições de movimentos nos } \\
\text { MMSS e de MMII. }\end{array}$ & $\begin{array}{l}\text { Queda significante da PAD e SP02 } \\
\text { entre o término da intervenção e } \\
\text { dois minutos após o término na } \\
\text { mobilização de MMSS. }\end{array}$ \\
\hline 4 & $\begin{array}{l}\text { COSTA } \\
\text { et al. (2019) }\end{array}$ & $\begin{array}{l}\text { Estudo de coor- } \\
\text { te prospectivo }\end{array}$ & $n=14$ & $\begin{array}{l}\text { GI: protocolo de MP de Morris, } \\
\text { com exercícios, transferências e } \\
\text { deambulação. }\end{array}$ & $\begin{array}{l}\text { Ganho de força muscular. GI apre- } \\
\text { sentou menor tempo de VMI e UTI } \\
\text { sem significância estatística. }\end{array}$ \\
\hline 5 & $\begin{array}{l}\text { COUTINHO } \\
\text { et al. (2016) }\end{array}$ & $\begin{array}{l}\text { Ensaio clínico } \\
\text { randomizado }\end{array}$ & $n=25$ & $\begin{array}{l}\text { GC: } 30 \text { min fisioterapia. } \\
\text { GI: realizou adicionalmente o } \\
\text { cicloergômetro por } 20 \mathrm{~min} \text {. }\end{array}$ & $\begin{array}{l}\text { Sem diferenças no tempo de VM, } \\
\text { de internação hospitalar e UTI. }\end{array}$ \\
\hline 6 & $\begin{array}{l}\text { EGGMANN } \\
\text { et al. (2018) }\end{array}$ & $\begin{array}{l}\text { Controlado } \\
\text { randomizado }\end{array}$ & $n=115$ & $\begin{array}{l}\text { GI: protocolo de treinamento de } \\
\text { resistência precoce e progressivo } \\
\text { combinado a MP de } 5 \text { a } 7 x \text { por } \\
\text { semana. }\end{array}$ & $\begin{array}{l}\text { Sem diferenças significativas } \\
\text { da independência e capacidade } \\
\text { funcional. }\end{array}$ \\
\hline 7 & $\begin{array}{l}\text { ESCALON } \\
\text { et al. }(2020)\end{array}$ & $\begin{array}{l}\text { Estudo de } \\
\text { coorte }\end{array}$ & $n=541$ & $\begin{array}{l}\text { Protocolo de mobilidade Monte } \\
\text { Sinai, iniciando a mobilização do } \\
\text { nível } 1 \text { com progressão até o nível } 4 .\end{array}$ & $\begin{array}{l}\text { Diminuição no tempo de perma- } \\
\text { nência na UTI e hospitalar, dias } \\
\text { em excesso, dias até o início da } \\
\text { fisioterapia. }\end{array}$ \\
\hline 8 & $\begin{array}{l}\text { FONTELA } \\
\text { et al. (2018) }\end{array}$ & $\begin{array}{l}\text { Estudo trans- } \\
\text { versal }\end{array}$ & $n=98$ & $\begin{array}{l}\text { MP foi definida como qualquer ati- } \\
\text { vidade realizada além da amplitude } \\
\text { de movimento, ocorrendo dentro de } \\
\text { 48h após o início da VM. }\end{array}$ & $\begin{array}{l}\text { Indisponibilidade de profissionais } \\
\text { da equipe, tempo insuficiente, } \\
\text { risco de auto lesão. }\end{array}$ \\
\hline 9 & $\begin{array}{l}\text { FONTELA } \\
\text { et al. (2018) }\end{array}$ & $\begin{array}{l}\text { Prospectivo, } \\
\text { observaciona, } \\
\text { multicêntrico, } \\
\text { de prevalência }\end{array}$ & $n=140$ & $\begin{array}{l}\text { Escala de mobilização de oito ní- } \\
\text { veis e duas categorias, permanecer } \\
\text { na cama (nível 1-3) e mobilizados } \\
\text { fora da cama (nível 4-8). }\end{array}$ & $\begin{array}{l}\text { Mobilização fora do leito aplicada } \\
\text { a apenas } 10 \% \text { dos pacientes. }\end{array}$ \\
\hline 10 & $\begin{array}{l}\text { HODGSON } \\
\text { et al. (2016) }\end{array}$ & $\begin{array}{l}\text { Piloto } \\
\text { Controlado } \\
\text { randomizado }\end{array}$ & $n=50$ & $\begin{array}{l}\text { GI: protocolo de MP direcionada a } \\
\text { objetivos, com atividades funcio- } \\
\text { nais ativas no período de } 30 \text { min a } 1 \\
\text { hora por dia. }\end{array}$ & $\begin{array}{l}\text { GI: níveis mais altos de atividade, } \\
\text { duração de exercícios ativos e } \\
\text { marcos de mobilidade. }\end{array}$ \\
\hline 11 & $\begin{array}{l}\text { LIU } \\
\text { et al. (2018) }\end{array}$ & $\begin{array}{l}\text { Estudo ob- } \\
\text { servacional } \\
\text { prospectivo }\end{array}$ & $n=232$ & $\begin{array}{l}\text { Protocolo de MP de Maebashi com } \\
\text { cinco níveis de reabilitação com } \\
\text { duração de } 20 \text { min. }\end{array}$ & $\begin{array}{l}62 \% \text { dos pacientes saíram da cama } \\
\text { em dois dias. }\end{array}$ \\
\hline 12 & $\begin{array}{l}\text { MACHADO } \\
\text { et al. (2017) }\end{array}$ & $\begin{array}{l}\text { Ensaio clínico } \\
\text { randomizado }\end{array}$ & $n=38$ & $\begin{array}{l}\text { GC: fisioterapia } 2 \mathrm{x} \text { ao dia por } \\
30 \mathrm{~min} \text {. GI utilizou adicionalmente } \\
\text { o cicloergômetro } 20 \mathrm{~min}, 5 \mathrm{x} \text { por } \\
\text { semana. }\end{array}$ & $\begin{array}{l}\text { Aumento da força muscular peri- } \\
\text { férica. Sem diferenças em relação } \\
\text { ao tempo de internação na UTI, } \\
\text { hospitalar e VM. }\end{array}$ \\
\hline 13 & $\begin{array}{l}\text { MARQUES FILHO } \\
\text { et al. (2018) }\end{array}$ & $\begin{array}{l}\text { Ensaio clínico } \\
\text { randomizado }\end{array}$ & $n=255$ & $\begin{array}{l}\text { G1:fisioterapia até } 12 \mathrm{~h} \text { depois da } \\
\text { admissão da UTI } \\
\text { G2: após } 12 \mathrm{~h} \text {. }\end{array}$ & $\begin{array}{l}\text { Houve redução do tempo de VM e } \\
\text { de hospitalização no G1. }\end{array}$ \\
\hline
\end{tabular}




\begin{tabular}{|c|c|c|c|c|c|}
\hline 14 & $\begin{array}{l}\text { MATOS } \\
\text { et al. }(2016)\end{array}$ & $\begin{array}{l}\text { Análise retros- } \\
\text { pectiva }\end{array}$ & $n=105$ & $\begin{array}{l}\text { Protocolo de MP de acordo com o } \\
\text { quadro clinico. Com a prescrição de } \\
\text { sentar fora do leito individualizada. }\end{array}$ & $\begin{array}{l}\text { Pacientes clínicos ficaram sentados } \\
\text { mais precocemente no leito. }\end{array}$ \\
\hline 15 & $\begin{array}{l}\text { MCWILLIAMS } \\
\text { et al. (2018) }\end{array}$ & $\begin{array}{l}\text { Controlado } \\
\text { randomizado } \\
\text { de viabilidade }\end{array}$ & $n=103$ & $\begin{array}{l}\text { GI: programa de reabilitação adap- } \\
\text { tado individualmente. }\end{array}$ & $\begin{array}{l}\text { GI: maior nível de mobilidade na } \\
\text { alta hospitalar e tempo de início } \\
\text { para a MP foi significativamente } \\
\text { menor. }\end{array}$ \\
\hline 16 & $\begin{array}{l}\text { SCHALLER } \\
\text { et al. (2016) }\end{array}$ & $\begin{array}{l}\text { Controlado } \\
\text { randomizado }\end{array}$ & $n=200$ & $\begin{array}{l}\text { GI: aplicado MP direcionada com } \\
\text { meta diária, do nível } 0 \text { ao } 4 \text {. }\end{array}$ & $\begin{array}{l}\text { GI atingiu níveis mais altos de } \\
\text { mobilização e seu tempo de per- } \\
\text { manência na UTI foi significativa- } \\
\text { mente menor. }\end{array}$ \\
\hline 17 & $\begin{array}{l}\text { SHOSHOLCHEVA } \\
\text { et al. (2016) }\end{array}$ & $\begin{array}{l}\text { Ensaio clínico } \\
\text { randomizado }\end{array}$ & $n=24$ & $\begin{array}{l}\text { G1: MP nas primeiras } 24-48 \mathrm{~h} \\
\text { G2: reabilitação posterior. }\end{array}$ & $\begin{array}{l}\text { Houve redução da duração de VM } \\
\text { no G1 sem diferença significativa. }\end{array}$ \\
\hline 18 & $\begin{array}{l}\text { SIBILLA } \\
\text { et al. }(2017)\end{array}$ & $\begin{array}{l}\text { Estudo de } \\
\text { prevalência }\end{array}$ & $n=161$ & $\begin{array}{l}\text { Pacientes com tubo endotraqueal, } \\
\text { traqueostomia ou VNI que realiza- } \\
\text { ram mobilização. }\end{array}$ & $\begin{array}{l}\text { Apenas } 33 \% \text { receberam mobiliza- } \\
\text { ção ativa. }\end{array}$ \\
\hline 19 & $\begin{array}{l}\text { SOUZA } \\
\text { et al. (2019) }\end{array}$ & $\begin{array}{l}\text { Estudo retros- } \\
\text { pectivo }\end{array}$ & $n=119$ & $\begin{array}{l}\text { GR: pacientes retirados do leito. } \\
\text { GM: pacientes mantidos no leito. }\end{array}$ & $\begin{array}{l}\text { Sem diferença significativa entre } \\
\text { os grupos no tempo de internação } \\
\text { e de VM. }\end{array}$ \\
\hline
\end{tabular}

Grupo controle (GC); grupo intervenção (GI); mantido no leito (GM); retirado do leito (GR); mobilização precoce (MP); pressão arterial diastólica (PAD); membros inferiores (MMII); membros superiores (MMSS); ventilação mecânica (VM); ventilação mecânica invasiva (VMI); saturação de oxigênio (SP02).

Fonte: Construção dos autores.

A Tabela 2 apresenta o resultado da avaliação de qualidade metodológica dos ensaios clínicos conforme critérios da escala PEDro, sendo analisado dez estudos, apresentando média de 6,4.

Tabela 2 - Avaliação da qualidade dos estudos através da escala PEDro.

\begin{tabular}{|c|c|c|c|c|c|c|c|c|c|c|c|c|}
\hline \multirow{2}{*}{ AUTORES } & \multicolumn{12}{|c|}{ Critérios da escala PEDro } \\
\hline & 1 & 2 & 3 & 4 & 5 & 6 & 7 & 8 & 9 & 10 & 11 & TOTAL \\
\hline Abruzzi, 2017 & $\mathrm{X}$ & - & - & - & - & - & - & - & - & - & - & 0 \\
\hline Bianchi, 2016 & $\mathrm{X}$ & - & - & $\mathrm{X}$ & - & - & - & $\mathrm{X}$ & $\mathrm{X}$ & $\mathrm{X}$ & $\mathrm{X}$ & 5 \\
\hline Coutinho, 2016 & $\mathrm{X}$ & $\mathrm{X}$ & - & $\mathrm{X}$ & - & - & - & $\mathrm{X}$ & $\mathrm{X}$ & $\mathrm{X}$ & $\mathrm{X}$ & 6 \\
\hline Eggmann, 2018 & $\mathrm{X}$ & $\mathrm{X}$ & $\mathrm{X}$ & $\mathrm{X}$ & $\mathrm{X}$ & $\mathrm{X}$ & - & - & $\mathrm{X}$ & $\mathrm{X}$ & $\mathrm{X}$ & 8 \\
\hline Hodgson, 2016 & $\mathrm{X}$ & $\mathrm{X}$ & $\mathrm{X}$ & $X$ & - & $\mathrm{X}$ & $X$ & $X$ & $\mathrm{X}$ & $\mathrm{X}$ & - & 8 \\
\hline Machado,2017 & $\mathrm{X}$ & $\mathrm{X}$ & $\mathrm{X}$ & $\mathrm{X}$ & $\mathrm{X}$ & $\mathrm{X}$ & $X$ & $\mathrm{X}$ & $\mathrm{X}$ & $\mathrm{X}$ & $\mathrm{X}$ & 10 \\
\hline Marques filho, 2018 & & $\mathrm{X}$ & - & $\mathrm{X}$ & - & - & - & $\mathrm{X}$ & $\mathrm{X}$ & $\mathrm{X}$ & $\mathrm{X}$ & 6 \\
\hline Mcwiliams, 2018 & $\mathrm{X}$ & $\mathrm{X}$ & $\mathrm{X}$ & $\mathrm{X}$ & - & - & - & - & $\mathrm{X}$ & $\mathrm{X}$ & $\mathrm{X}$ & 6 \\
\hline Schaller, 2016 & $\mathrm{X}$ & $\mathrm{X}$ & $\mathrm{X}$ & $\mathrm{X}$ & $\mathrm{X}$ & $\mathrm{X}$ & - & $\mathrm{X}$ & $\mathrm{X}$ & $\mathrm{X}$ & $\mathrm{X}$ & 9 \\
\hline Shosholcheva, 2016 & $\mathrm{X}$ & $\mathrm{X}$ & - & $\mathrm{X}$ & - & - & - & $\mathrm{X}$ & $\mathrm{X}$ & $\mathrm{X}$ & $\mathrm{X}$ & 6 \\
\hline Média & & & & & & & & & & & & 6,4 \\
\hline
\end{tabular}

Fonte: Construção dos autores.

\section{DISCUSSÃO}

O objetivo deste estudo foi sintetizar as evidências científicas da eficácia da mobilização precoce em pacientes internados em UTI com o uso de ventilação mecânica por meio de revisão bibliográfica. A FAUTI é uma complicação frequentemente encontrada na UTI, pois apresenta como fator 
de risco o imobilismo. Com a MP, os autores buscaram reduzir esses risco para que os pacientes apresentem o mínimo possível de complicações quando estão na UTI em uso de VM, como é o caso do estudo de Eggmann et al. (2018), que realizou um treino de resistência combinado com a MP, resultando em uma melhora na capacidade e independência funcional do paciente do grupo intervenção, aumento da força muscular e alta hospitalar precoce; e o estudo de Schaller et al. (2016), que utilizou a MP em pacientes cirúrgicos, onde obteve melhora da capacidade funcional e diminuição do tempo de internação na UTI, visando minimizar os efeitos deletérios do imobilismo.

Segundo o estudo realizado por Hodgson et al. (2016), um protocolo de MP para pacientes que estavam em VM com tempo mínimo de 24 horas apresentou inúmeros benefícios, e a intervenção é similar aos achados em outros dois estudos, onde o primeiro propõe um ensaio de mobilização muito precoce (AVERT) em pacientes com AVE agudo e apresentou resultados favoráveis à aplicação, sendo uma mobilização de curta duração e mais frequente (LANGHORNE et al., 2017). Os achados neste estudo corroboram com o de Rethnam et al. (2020), onde também realizou intervenção de mobilização nas primeiras 20 horas pós AVE, com resultados positivos.

Conforme estudo realizado por Abruzzi et al. (2017) e Marques Filho et al. (2018), ambos apresentaram resultados semelhantes, onde os pacientes que iniciaram o tratamento em um tempo inferior a 24 horas apresentaram maiores benefícios, como redução do tempo de internação e diminuição da duração de VM. Entretanto, em relação ao grupo que realizou o tratamento após as 24 horas, concluíram que a MP quando iniciada em um período de tempo menor se torna benéfica, segura e viável aos pacientes.

De acordo com dois estudos, Souza et al. (2019) e Godoy et al. (2015), estes, ao realizar uma análise de prontuários, não observaram diferenças significativas entre o tempo de internação na UTI comparado ao uso de VM. Entretanto, os achados referentes a pacientes mantidos e retirados do leito, obteve-se uma diferença significativa. Esses dados são semelhantes ao estudo de Matos et al. (2016), que realizou um protocolo para pacientes clínicos e cirúrgicos, afirmando que os pacientes clínicos obtiveram resultados significativamente melhores ao se sentarem fora do leito do que pacientes cirúrgicos.

Conforme os estudos que utilizaram o cicloergômetro na reabilitação precoce dos pacientes críticos internados na UTI com VM, concluíram que cicloergômetro possui vários benefícios, auxiliando na doença pulmonar obstrutiva crônica, autopercepção funcional, aumento da força de quadríceps e da capacidade funcional do paciente. O estudo de Coutinho et al. (2016) resultou em diferenças estatísticas não significativas na hemodinâmica dos pacientes e na mecânica respiratória, sem alterações fisiológicas. Bianchi et al. (2016) analisou a mobilidade diafragmática dos pacientes e identificou que em ambos os grupos a mobilidade se manteve. Machado et al. (2017) relatou em seu trabalho que os pacientes do grupo intervenção apresentaram aumento da força muscular, porém, este resultado não teve relação com a diminuição do tempo de internação hospitalar e de VM. 
Seis estudos analisados (COSTA et al., 2019; HODGSON et al., 2016; LIU et al., 2018; ESCALON et al., 2020; MCWILLIAMS et al. 2018; CAVALCANTE et al., 2018) descreveram a implementação de um programa de MP através de protocolos padronizados. A maioria dos estudos apresentavam um protocolo semelhante e resultados positivos e eficazes, sendo os principais o aumento da força muscular, diminuição do suporte ventilatório, diminuição do tempo de internação na UTI e aumento da duração de realização dos exercícios ativos e de mobilidade. Apenas um estudo, de Cavalcante et al., (2018), apresentou um protocolo distinto, utilizando apenas a MP passiva, onde não foi possível identificar diferenças significativas entre o grupo intervenção e o grupo controle.

Os fatores hemodinâmicos são fundamentais para que se possa realizar e evoluir na MP, e três estudos observaram se a MP alterou esses fatores. Como resultado, foi verificado que os pacientes apresentaram melhora na frequência respiratória, gasometria arterial, temperatura corporal, oxigenação, escala de Glasgow (SHOSHOLCHEVA et al., 2016), diminuição da pressão arterial diastólica, saturação, aumento da pressão arterial sistólica (CAVALCANTE et al., 2018) e também foi encontrado diferença significativa do bicarbonato na troca gasosa pela gasometria arterial(COUTINHO et al., 2016).

Alguns dos estudos analisados tinham como objetivo analisar as barreiras encontradas com a utilização da MP em pacientes internados em UTI. No estudo realizado por Fontela et al. (2018) observou-se que as principais barreiras encontradas eram a sedação, fraqueza e a instabilidade cardiovascular, sendo que $90 \%$ dos pacientes foram mobilizados apenas no leito. Resultados semelhantes com outros estudos (SIBILLA et al., 2017; FONTELA et al., 2018) destacaram as contraindicações médicas e a instabilidade cardiovascular como barreira e a realização da MP na UTI como desafiadora, devido ao tempo insuficiente, indisponibilidade da equipe, excesso de sedação, delirium, risco de auto lesão musculoesquelética e excesso de estresse no trabalho.

Dessa forma, podemos destacar que muitos dos estudos analisados por esta pesquisa apontam que a MP apresenta benefícios para pacientes em uso de VM, mas algumas limitações foram encontradas, como a prevalência da MP ser considerada baixa na maioria dos hospitais e a falta de disponibilidade dos profissionais com experiência na área intensiva.

\section{CONCLUSÃO}

A partir dos resultados analisados, observamos evidências de que a MP é uma técnica segura e eficaz na redução do tempo de internação e tempo de VM em pacientes críticos, melhorando a força e resistência muscular com possibilidades baixas de eventos adversos, apresentando resultados satisfatórios quando realizada antes das 24 horas e se desenvolvida com progressão em diferentes níveis de mobilidade. Não havendo diferenças entre o uso apenas da MP ou com associação ao cicloergômetro. Entretanto, sua utilização na UTI apresenta algumas barreiras, como o excesso de sedação, instabilidade cardiovascular, tempo reduzido e a indisponibilidade de profissionais com experiência em 
terapia intensiva. Este estudo contém algumas limitações como a inclusão de diferentes tipos de estudos devido à escassez de estudos de intervenção, sendo que alguns estudos incluídos apresentaram baixa qualidade metodológica analisada pela escala de PEDro. Dessa forma, sugere-se a necessidade de novos estudos com maior rigor metodológico.

\section{REFERÊNCIAS}

ABRUZZI, F. et al. Ultra early mobilization reduces the time of mechanical ventilation and ICU stay. Critical Care, v. 21, n. 1, 2017. Acesso em: 28 jul. 2020.

AQUIM, E. E. et al. Brazilian guidelines for early mobilization in intensive care unit. Revista Brasileira de Terapia Intensiva, v. 31, n. 4, p. 434-443, 2019.Acesso em: 02 ago. 2020.

BIANCHI, T. Efeito do cicloergômetro passivo sobre a mobilidade diafragmática de pacientes críticos em ventilação mecânica invasiva na unidade de terapia intensiva: ensaio clínico randomizado. BioMed Central, v. 16, 2016. Acesso em: 28 jul. 2020.

CAVALCANTE, E. A. F. P. et al. Repercussões da mobilização passiva nas variáveis hemodinâmicas em pacientes sob ventilação mecânica. Journal of Health \& Biological Sciences, v. 6, n. 2, p. 170, 2018. Acesso em: 28 jul. 2020.

COSTA, C. C.; LEITE, S.; FORTINO, C. K. Evaluation of an early mobilization protocol in a unit of intensive therapy. Revista Conhecimento Online, v. 3, p. 92-114, 2019. Acesso em: 03 ago. 2020.

COUTINHO, W. M. et al. Efeito agudo da utilização do cicloergômetro durante atendimento fisioterapêutico em pacientes críticos ventilados mecanicamente. Fisioterapia e Pesquisa, v. 23, n. 3, p. 278-283, 2016. Acesso em: 28 jul. 2020.

DANTAS, C. M. et al. Influência da mobilização precoce na força muscular periférica e respiratória em pacientes críticos. Revista Brasileira de Terapia Intensiva, v. 24, n. 2, p. 173-178, 2012. Acesso em: 28 jul. 2020.

EGGMANN, S. et al. Effects of early, combined endurance and resistance training in mechanically ventilated, critically ill patients: A randomised controlled trial. PLoS ONE, v. 13, n. 11, p. 1-19, 2018. Acesso em: 28 jul. 2020. 
ESCALON, M. X. et al. The Effects of Early Mobilization on Patients Requiring Extended Mechanical Ventilation Across Multiple ICUs. Critical Care Explorations, v. 2, n. 6, p. e0119, 2020. Acesso em: 29 jul. 2020.

FONTELA, P. C.; FORGIARINI, L. A.; FRIEDMAN, G. Clinical attitudes and perceived barriers to early mobilization of critically ill patients in adult intensive care units. Revista Brasileira de Terapia Intensiva, v. 30, n. 2, p. 187-194, 2018. Acesso em: 28 jul. 2020.

FONTELA, P. C. et al. Early mobilization practices of mechanically ventilated patients: a 1-day point-prevalence study in southern Brazil. Clinics, São Paulo, v. 73, n. 23, p. e241, 2018. Acesso em: 28 jul. 2020.

GALVÃO, T. F.; PANSANI, T. S. A.; HARRAD, D. Principais itens para relatar Revisões sistemáticas e Meta-análises: A recomendação PRISMA. Epidemiologia e Serviços de Saúde, v. 24, n. 2, p. 335-342, 2015. Acesso em: 02 ago. 2020.

GODOY, M. D. P. et al. Fraqueza muscular adquirida na UTI (ICU-AW): efeitos sistêmicos da eletroestimulação neuromuscular. Revista Brasileira de Neurologia, v. 51, n. 4, p. 110-113, 2015. Acesso em: 02 ago. 2020.

HERMANS, G.; VAN DEN BERGHE, G. Clinical review: Intensive care unit acquired weakness. Critical Care, v. 19, n. 1, p. 1-9, 2015. Disponível em: http://dx.doi.org/10.1186/s13054-015-0993-7. Acesso em: 28 jul. 2020.

HODGSON, C. L. et al. A binational multicenter pilot feasibility randomized controlled trial of early goal-directed mobilization in the ICU. Critical Care Medicine, v. 44, n. 6, p. 1145-1152, 2016. Acesso em: 28 jul. 2020.

KRAMER, C. L. Intensive Care Unit-Acquired Weakness. Neurologic Clinics, v. 35, n. 4, p. 723-736, 2017. Elsevier Inc. Disponível em: https://doi.org/10.1016/j.ncl.2017.06.008. Acesso em: 28 jul. 2020.

LANGHORNE, P. et al. A very early rehabilitation trial after stroke (AVERT): a Phase III, multicentre, randomised controlled trial. Health Technology Assessment, v. 21, n. 54, p. 1-119, 2017. Acesso em: 02 ago. 2020. 
LIU, K. et al. The safety of a novel early mobilization protocol conducted by ICU physicians: A prospective observational study. Journal of Intensive Care, v. 6, n. 1, p. 1-11, 2018. Acesso em: 03 ago. 2020.

MACHADO, A. dos S. et al. Efeito do exercício passivo em cicloergômetro na força muscular, tempo de ventilação mecânica e internação hospitalar em pacientes críticos: ensaio clínico randomizado. Jornal Brasileiro de Pneumologia, v. 43, n. 2, p. 134-136, 2017. Acesso em: 29 jul. 2020.

MARQUES FILHO, P. R. et al. Prevalence of early mobilization and its effects within the intensive care unit. Intensive Care Medicine Experimental, v. 6, n. 40, 2018. Acesso em: 28 jul. 2020.

MATOS, C. A. de et al. Existe diferença na mobilização precoce entre os pacientes clínicos e cirúrgicos ventilados mecanicamente em UTI? Fisioterapia e Pesquisa, v. 23, n. 2, p. 124-128, 2016. Acesso em: 04 ago. 2020.

MCWILLIAMS, D. et al. Earlier and enhanced rehabilitation of mechanically ventilated patients in critical care: A feasibility randomised controlled trial. Journal of Critical Care, v. 44, p. 407-412, 2018. Disponível em: https://doi.org/10.1016/j.jcrc.2018.01.001. Acesso em: 28 jul. 2020.

RETHNAM, V. et al. Early mobilisation post-stroke: a systematic review and meta-analysis of individual participant data. Disability and Rehabilitation, p. 1-8, 2020. Acesso em: 04 ago. 2020.

ROTTA, B. P. et al. Relação entre a disponibilidade de serviços de fisioterapia e custos de UTI. Jornal Brasileiro de Pneumologia, v. 44, n. 3, p. 184-189, mar. 2018.Acesso em: 02 ago. 2020.

SCHALLER, S. J. et al. Early, goal-directed mobilisation in the surgical intensive care unit: a randomised controlled trial. The Lancet, v. 388, n. 10052, p. 1377-1388, 2016. Disponível em: http://dx.doi.org/10.1016/ S0140-6736(16)31637-3. Acesso em: 29 jul. 2020.

SCHUJMANN, D. S. et al. Impact of a progressive mobility program on the functional status, respiratory, and muscular systems of ICU patients: A randomized and controlled trial. Critical Care Medicine, v. 48, n. 4, p. 491-497, 2019. Acesso em: 29 jul. 2020.

SHOSHOLCHEVA, M. et al. Early physical rehabilitation improves outcome at mechanical ventilated patients. Anesthesia \& Analgesia, v. 123, n. 3, p. 168, 2016. Acesso em: 29 jul. 2020. 
SIBILLA, A. et al. Mobilization of Mechanically Ventilated Patients in Switzerland. Journal of

Intensive Care Medicine, v. 35, n. 1, p. 55-62, 2017.Acesso em: 28 jul. 2020.

SLUTSKY, A. S. History of Mechanical Ventilation. From Vesalius to Ventilator-induced Lung Injury. American journal of respiratory and critical care medicine, v. 191, n. 10, p. 1106-1115, 2015. Acesso em: 29 jul. 2020.

SOUZA, T. S. de et al. Relação entre a retirada do leito com tempo de ventilação mecânica invasiva e tempo de internação na uti. Revista Inspirar, v. 19, n. 2, p. 1-14, 2019. Acesso em: 29 jul. 2020.

ZANG, K. et al. The effect of early mobilization in critically ill patients: A meta-analysis. Nursing in Critical Care, v.2. n.1. May, p. 1-8, 2018. Acesso em: 28 jul. 2020. 